\title{
CONTENT BASED IMAgE RETRIEVAL (CBIR) USING MULTIPLE FEATURES For TEXTILE IMAGES BY USING SVM CLASSIFIER
}

\author{
Mr.P.Anand ${ }^{1}$ (AP/ECE), T.Ajitha ${ }^{2}$, M.Priyadharshini ${ }^{3}$ and M.G.Vaishali ${ }^{4}$ \\ Velammal college of Engineering and Technology, Madurai-09.
}

\begin{abstract}
In this project, we proposed a Content Based Image Retrieval (CBIR) system which is used to retrieve a relevant image from an outsized database. Textile images showed the way for the development of CBIR. It establishes the efficient combination of color, shape and texture features. Here the textile image is given as dataset. The images in database are loaded. The resultant image is given as input to feature extraction technique which is transformation of input image into a set of features such as color, texture and shape. The texture feature of an image is taken out by using Gray level co-occurrence matrix (GLCM). The color feature of an image is obtained by HSI color space. The shape feature of an image is extorted by sobel technique. These algorithms are used to calculate the similarity between extracted features. These features are combined effectively so that the retrieval accuracy and recall rate is enhanced. The classification techniques such as Support Vector Machine (SVM) are used to classify the features of a query image by splitting the group such as color, shape and texture. Finally, the relevant images are retrieved from a large database and hence the efficiency of an image is plotted.The software used is MATLAB 7.10 (matrix laboratory) which is built software applications
\end{abstract}

\section{KEYWORDS}

CBIR, HSI color space, SVM, Feature extraction.

\section{I.INTRODUCTION:}

The course of textile images plays a vital role in content based image retrieval (CBIR). Thus the task is capably storing, processing and retrieving textile image records. "Content-based" means that the search will analyze the actual contents of the image rather than the metadata such as keywords, tags, and/or descriptions associated with the image. The term 'content' in this context might refer to colors, shapes, textures, or any other information that can be derived from the image metadatass itself. Our proposed work is mentioned as steps given below.

Step 1: Loading image

a). Convert the image into workable format with the extension as .bmp or .jpg.

b). Convert the workable format into gray scale image.

Step 2: Feature Extraction

a). The texture, color and shape features are extracted gray level co-occurrence matrix, HSI color space, sobel technique respectively.

b). Make the feature vector by making the average value of each pixels byte.

Step 3: Similarity computation

a). Compute the similarity on the basis of distance above and below the threshold value.

Step 4: Mark the relevant and irrelevant images.

DOI : 10.5121/ijcsity.2014.2204 
Step 5: Classify the image set.

Step 6: Train the SVM classifier.

Section II details about Block Diagram. Section III describes about Feature Extraction Techniques. Section IV explained about an Optimization techniques.

\section{II.BLOCK DIAGRAM}

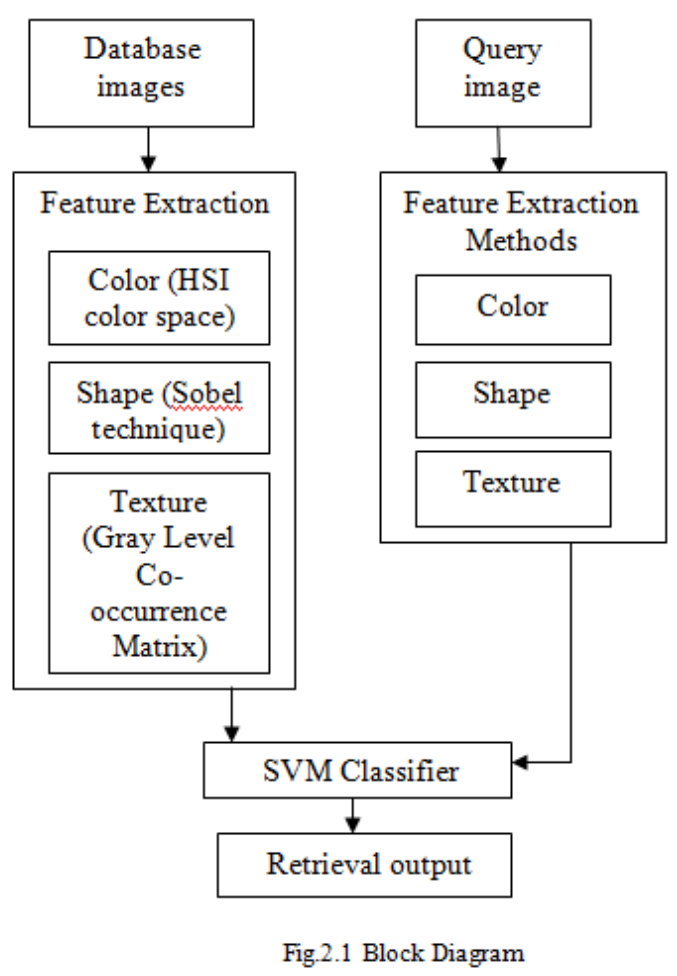

\section{FEATURE EXTRACTION}

In this project three features are extracted by using appropriate techniques. Color features are extracted using HSI color space, shape features are extracted using sobel technique and texture features are extracted using gray level co-occurrence matrix. These features are explained below.

\section{Color feature extraction:}

Textile image is given as a query input image. For each and every image the RGB color space is converted into HSI color space. Then the single image is partitioned into three regions namely Hue, Saturation and Intensity. The properties of color such as mean, standard deviation and skewness are determined only for hue color space image. These properties are described below.

\section{A) Mean:}

Mean is defined as the average value of a color in an image. The mean of an image is considered as a first central moment. The mean value is calculated for each region by using the formula given below. 
International Journal of Computational Science and Information Technology (IJCSITY) Vol.2, No.2, May 2014

$$
\mathrm{Ei}=\sum_{N}^{j=1} \frac{1}{N} \mathrm{Pij}
$$

\section{B) Standard deviation:}

The standard deviation is defined as the square root of the variance of distribution. The standard deviation of an image is considered as a second central moment. The standard deviation value is calculated for each region by using the formula given below.

$$
\sigma_{\mathrm{i}=} \sqrt{ }\left(\frac{1}{N} \sum_{N}^{j=1} A\right)
$$

Where, $\mathrm{A}=(\mathrm{Pij}-\mathrm{Ei}) 2$

\section{C) Skewness:}

Skewness is defined as a measure of the degree of asymmetry in the distribution. The skewness of an image is considered as a third central moment.

$$
\mathrm{Si}=\sqrt[3]{ }\left(\frac{1}{N} \sum_{N}^{j=1} B\right)
$$

Where, $B=(P i j-E i)^{3}$

The skewness value is calculated for each region by using the formula given above.

Where,

I is the current channel index (ie) $\mathrm{H}=1, \mathrm{~S}=2, \mathrm{~V}=3$

Ei1, Ei2 are the first moment (mean) of two image.

$\sigma \mathrm{i}$ is the second moment (standard deviation) of two image distribution.

$\mathrm{Si}$ is the third moment (skewness) of two image distribution.

The values are obtained as follows.

\begin{tabular}{|l|l|l|l|}
\hline Images & Mean & $\begin{array}{l}\text { Standard } \\
\text { deviation }\end{array}$ & Skewness \\
\hline 1.jpg & 0.3483 & 0.2467 & -0.1130 \\
\hline 2.jpg & 0.4006 & 0.2663 & -0.7362 \\
\hline 3.jpg & 0.3708 & 0.2730 & -0.5192 \\
\hline 4.jpg & 0.4090 & 0.3240 & -0.4316 \\
\hline 5.jpg & 0.0353 & 0.1461 & 5.3085 \\
\hline 6.jpg & 0.4191 & 0.3021 & -0.5950 \\
\hline 7.jpg & 0.5053 & 0.4396 & -0.2396 \\
\hline 8.jpg & 0.4111 & 0.2186 & -0.6466 \\
\hline 9.jpg & 0.4500 & 0.2646 & -0.9391 \\
\hline 10.jpg & 0.3712 & 0.2175 & -0.1779 \\
\hline 11.jpg & 0.3543 & 0.2976 & -0.2670 \\
\hline 12.jpg & 0.3950 & 0.2826 & -0.2002 \\
\hline 13.jpg & 0.6765 & 0.4343 & -0.8275 \\
\hline 14.jpg & 0.2978 & 0.2787 & -0.0621 \\
\hline 15.jpg & 0.5172 & 0.4638 & -0.1682 \\
\hline
\end{tabular}

Table.3.1 Color Feature Values 


\section{Shape Feature Extraction}

The input image is converted into gray scale image and applying Sobel technique for edge preservation. Eccentricity and centroid are the properties focused on shape feature extraction. These features are explained below.

\section{A) Eccentricity:}

It is the ratio of major axis to minor axis. It characterizes the length of ROI. Lateral mean and longitudinal mean values are calculated for eccentricity. The eccentricity is obtained by using the formula given below.

$$
\epsilon=((\mu 20-\mu 02) 2+4 \mu 112) /(\mu 20+\mu 02) 2
$$

\section{B) Centroid:}

The point at the centre of image called centre of area. The coordinates of centroid are the average (arithmetic mean) of the coordinates of points in image. The centroid linkage uses the Euclidean distance between the centroid of two clusters.

$$
\mathrm{d}(\mathrm{r}, \mathrm{s})=\|\mathrm{Xr}-\mathrm{Xs}\| 2
$$

The values are listed in table 3.2

\begin{tabular}{|l|l|l|l|l|}
\hline Images & $\begin{array}{l}\text { Lat } \\
\text { mean1 }\end{array}$ & $\begin{array}{l}\text { Lat } \\
\text { mean2 }\end{array}$ & $\begin{array}{l}\text { Lon } \\
\text { mean1 }\end{array}$ & $\begin{array}{l}\text { Lon } \\
\text { mean2 }\end{array}$ \\
\hline 1.jpg & 0.5328 & 0.5481 & 0.5034 & 0.5340 \\
\hline 2.jpg & 0.5119 & 0.5025 & 0.5184 & 0.5029 \\
\hline 3.jpg & 0.5249 & 0.5281 & 0.4900 & 0.5179 \\
\hline 4.jpg & 0.5073 & 0.5201 & 0.4949 & 0.4969 \\
\hline 5.jpg & 0.5066 & 0.4749 & 0.5032 & 0.5005 \\
\hline 6.jpg & 0.5479 & 0.5268 & 0.5187 & 0.4970 \\
\hline 7.jpg & 0.5145 & 0.5334 & 0.5008 & 0.5109 \\
\hline 8.jpg & 0.5000 & 0.5305 & 0.5847 & 0.4654 \\
\hline 9.jpg & 0.5267 & 0.4968 & 0.5182 & 0.4817 \\
\hline 10.jpg & 0.5276 & 0.5191 & 0.5019 & 0.5116 \\
\hline 11.jpg & 0.4966 & 0.5129 & 0.4588 & 0.4654 \\
\hline 12.jpg & 0.5139 & 0.5331 & 0.4807 & 0.5024 \\
\hline 13.jpg & 0.5218 & 0.5348 & 0.5128 & 0.5054 \\
\hline 14.jpg & 0.5216 & 0.4998 & 0.4984 & 0.5030 \\
\hline 15.jpg & 0.5480 & 0.5363 & 0.4992 & 0.4967 \\
\hline
\end{tabular}

Table.3.2 Shape Feature Values

\section{Texture Feature Extraction:}

The texture features are extracted by using Gray Level Co-occurrence Matrix (GLCM). It is used to calculate how often pixels with gray level value $\mathrm{i}$ occurs horizontally adjacent to a pixel with a value $\mathrm{j}$. Finding co-occurrence matrix is to remove redundancy. The features extracted are Energy, Entropy, Contrast and Correlation. These values are obtained by using formula. Matching is done with query image and database images. 
International Journal of Computational Science and Information Technology (IJCSITY) Vol.2, No.2, May 2014

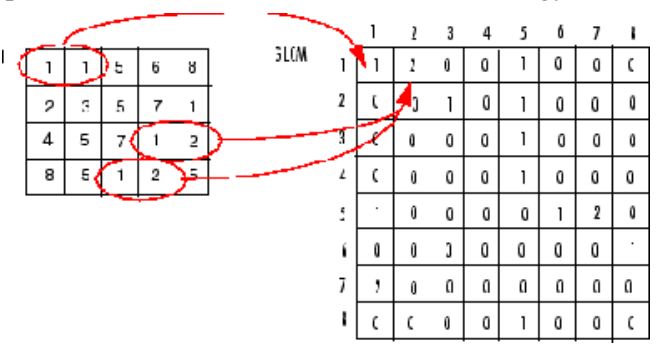

Fig 3.3 GLCM

\section{A) Contrast:}

It measures local variations in gray level co-occurrence matrix. It returns a measure of intensity contrast between a pixel and its neighbor over the whole image. The formula for contrast is given below.

\section{B) Correlation:}

$$
\sum_{i, j}|i-j|^{2} p(i, j)
$$

It measures joint probability occurrence of specified pixel pairs. It returns a measure of how correlated a pixel to its neighbor of over whole image. The range lies within [-1,1]. Correlation is 1 for positively image. It's -1 for negatively image. Nan is for constant image.

$$
\sum_{i, j} \frac{(i-\mu i)(j-\mu j) p(i, j)}{\sigma_{i} \sigma_{j}}
$$

\section{C) Energy:}

It provides the sum of squared elements in GLCM known as uniformity or angular second moments. Range lies within range [0 1].

Energy is 1 for constant image.

\section{D) Homogeneity:}

$$
\sum_{i, j} p(i, j)^{2}
$$

It measures closeness of distribution of elements in GLCM to GLCM diagonal. Returns a value that measures the closeness of the distribution of elements in the GLCM to the GLCM diagonal. Homogeneity is 1 for diagonal matrix. The range lies within [0 1].

The values are listed in table.3.4

$$
\sum_{i, j} \frac{p(i, j)}{1+|i-j|}
$$


International Journal of Computational Science and Information Technology (IJCSITY) Vol.2, No.2, May 2014

\begin{tabular}{|l|l|l|l|l|}
\hline Images & Contrast & Correlation & Energy & Homogeneity \\
\hline 1.jpg & 0.1373 & 0.9767 & 0.3177 & 0.9677 \\
\hline 2.jpg & 0.0921 & 0.9768 & 0.4960 & 0.9823 \\
\hline 3.jpg & 0.1568 & 0.9657 & 0.4294 & 0.9708 \\
\hline 4.jpg & 0.5820 & 0.9666 & 0.4684 & 0.9727 \\
\hline 5.jpg & 0.1390 & 0.9691 & 0.4653 & 0.9785 \\
\hline 6.jpg & 1.6310 & 0.8339 & 0.2140 & 0.8266 \\
\hline 7.jpg & 0.3134 & 0.9713 & 0.3147 & 0.9534 \\
\hline 8.jpg & 0.1393 & 0.8427 & 0.3432 & 0.9317 \\
\hline 9.jpg & 0.4422 & 0.9714 & 0.3169 & 0.9454 \\
\hline 10.jpg & 0.4132 & 0.9619 & 0.3201 & 0.9395 \\
\hline 11.jpg & 0.3396 & 0.9734 & 0.4283 & 0.9712 \\
\hline 12.jpg & 0.2585 & 0.9166 & 0.3067 & 0.9548 \\
\hline 13.jpg & 0.4239 & 0.9556 & 0.2464 & 0.9131 \\
\hline 14.jpg & 0.3988 & 0.9603 & 0.3256 & 0.9435 \\
\hline 15.jpg & 0.4625 & 0.9693 & 0.2569 & 0.8940 \\
\hline
\end{tabular}

Table.3.4 Texture Feature Values

\section{SUPPORT VECTOR MACHINE (SVM):}

SVM is suitable to classify the Color and shape features accurately and efficiently. It is superintend learning process which analyze and recognize patterns such as texture, color, shape and it is used for classification and regression techniques. The classification is accomplished by hyper plane which has outsized distance to nearest distance training data points of any class (color, texture, shape). Therefore the observation is larger the margin, lower the generalization error of classifier. The classification separates data into training samples and testing samples. The objective of SVM is to predict the target values of test data by giving only the test data attributes.

The steps are to be followed:

1. Transform the data to the format of SVM which is suitable for further process.

2. Applying scaling on data.

3. Consider the RBF kernel function

$$
\mathrm{K}(\mathrm{x} ; \mathrm{y})=\exp (-\alpha\|\mathrm{x}-\mathrm{y}\| 2)
$$

4. By using the best parameter $\mathrm{C}$, cross validation to train the entire training set.

5. Finally testing is needed to check the error is within acceptable limit.

It is a non parametric study with binary classifier. It is efficient because it accepts more input variables. It only depends upon selection of hyper plane and kernel parameter.

\section{V) RESULTS AND DISCUSSION:}

Initially the features of color, shape, texture for textile images extracted are tabulated. The fig.5.1 shows that the given input image is textile T-Shirt image. The input RGB image is converted into 
International Journal of Computational Science and Information Technology (IJCSITY) Vol.2, No.2, May 2014 hue image. The color features are extracted using HSI color space. The output is displayed as Hue image.
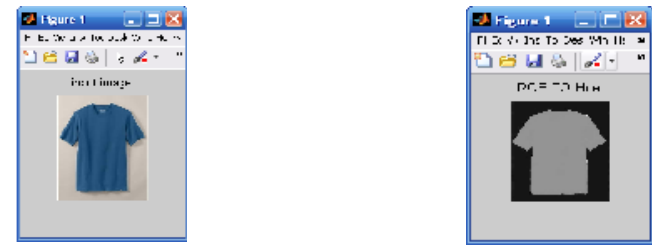

Fig.5.1 Color Feature Extraction

The given input textile image is displayed as shown in fig.5.2. The sobel technique is applied to given input image. The edge is preserved by calculating the gradients. The preserved edge is shown below in fig.5.2.
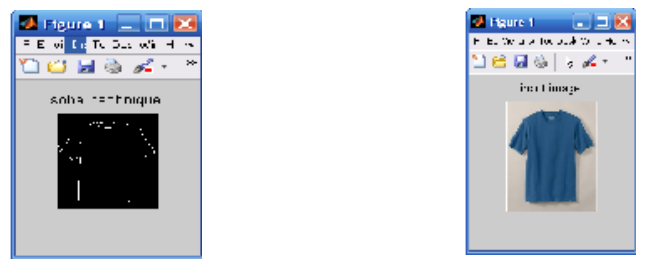

Fig.5.2 Shape Feature Extraction

The given input image is displayed in fig.5.3. The given RGB image is converted into gray scale image. The texture features are extracted using Gray level Co-Occurrence matrix. The output image is displayed in fig.5.3.
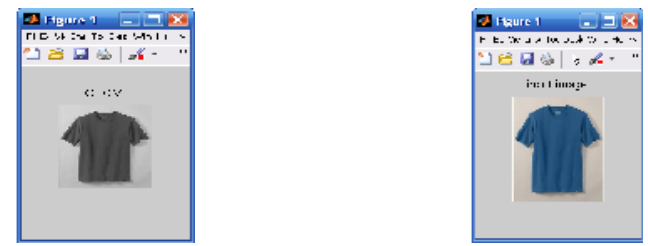

Fig.5.3 Texture Feature Extraction

The three features such as color, texture, shape are extracted. The features are fed into SVM classifier. The query image is sent and the three features are extracted similarly and fed to classifier.

The classifier output is described below in fig.5.4.1 the color feature extracted SVM output.

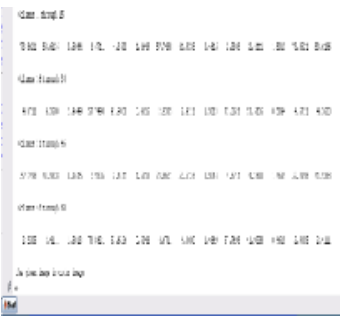

Fig.5.4.1. The color feature extracted SVM output 
International Journal of Computational Science and Information Technology (IJCSITY) Vol.2, No.2, May 2014

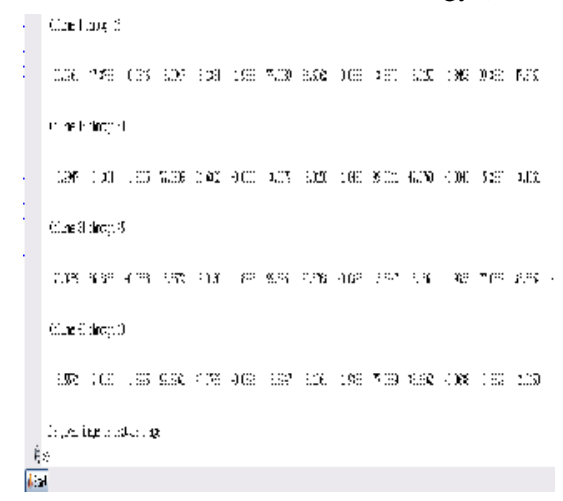

Fig.5.4.2.The texture feature extracted SVM output.

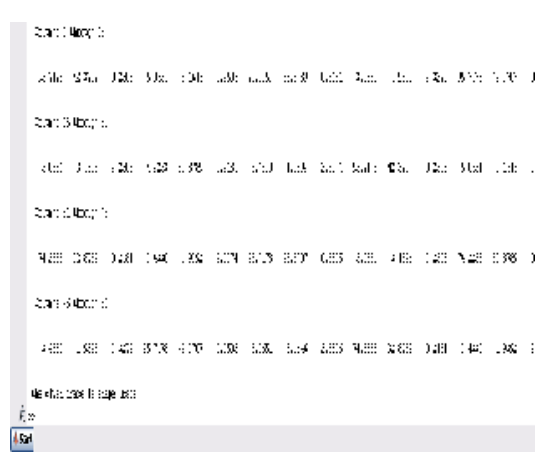

Fig.5.4.3.The Shape feature extracted SVM output.

Finally the similar images are retrieved. and then compare the accuracy and retrieval time in our project.

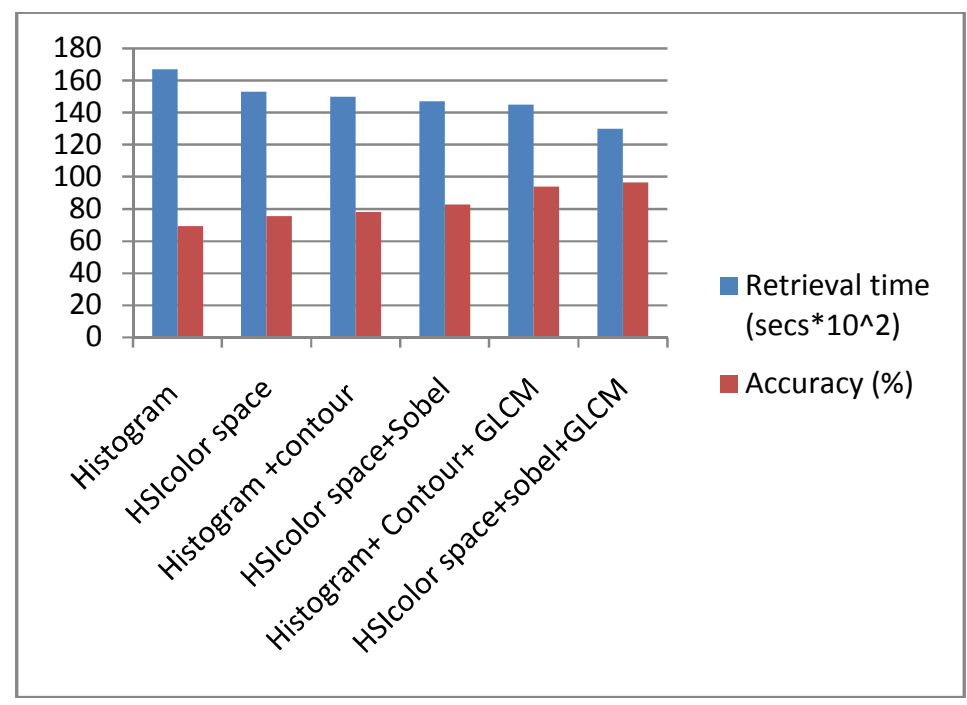

Fig.5.4 compared accuracy for proposed work and existing work 
International Journal of Computational Science and Information Technology (IJCSITY) Vol.2, No.2, May 2014

\section{REFERENCES:}

[1] Kodituwakku S. R, Selvarajah S. "Comparison of Color Features for Image Retrieval”. Indian Journal of Computer Science and Engineering, Vol. 1, No. 3, pp: 207-211, 2010.

[2] Felci Rajam I. and Valli S. "SRBIR: semantic region based image retrieval by extracting the dominant region and semantic learning”. Journal of Computer Science, Vol. 7, No. 3, pp.400-408, 2011 a.

[3] Felci Rajam I and Valli S. "Content-Based Image Retrieval Using a Quick SVM-Binary Decision Tree - QSVMBDT”. Springer Communications in Computer and Information Science 205, pp: 11-22, $2011 b$.

[4] FelciRajam I. and Valli S. "Region-based image retrieval using the semantic cluster matrix and adaptive learning". International Journal of Computational Science and Engineering, Vol. 7, No. 3, pp.239-252, 2012.

[5] Celia B, Felci Rajam I. "An efficient content based image retrieval framework using machine learning techniques". Proceedings of the Second international conference on Data Engineering and Management (ICDEM 10), Springer LNCS, Vol. 6411, pp 162-169, 2010.

[6] Lining Zhang, Lipo Wang andWeisi Lin. "Generalized Biased Discriminant Analysis for ContentBased Image Retrieval”. IEEE Transactions on Systems, Man, and Cybernetics-Part B: Cybernetics, Vol. 42, No. 1, pp. 282-290, 2012.

[7]I mtnan-Ul-Haque Qazi, OlivierAlata, Jean-ChristopheBurie, Ahmed Moussa, ChristineFernandezMaloigne. "Choice of a pertinent color space for color texture characterization using parametric spectral analysis”. Pattern Recognition 44, pp. 16-31, 2011.

[8] Hatice Cinar Akakin and Metin N. Gurcan. "Content-Based Microscopic Image Retrieval System for Multi-Image queries”. IEEE Transactions on Information Technology in Biomedicine, Vol. 16, No. 4, pp. $758-769,2012$.

[9] WangXing-yuan, ChenZhi-feng, YunJiao-jiao. "An effective method for color image retrieval based on texture". Computer Standards \& Interfaces 34, pp. 31- 35, 2012.

[10] Samuel Rota Bul_o, Massimo Rabbi and Marcello Pelillo. "Content-Based Image Retrieval with Relevance Feedback using Random Walks”. Pattern Recognition, Vol. 44, No. 9, pp. 2109-2122, 2011.

[11] Neetu Sharma S, Paresh Rawat S and jaikaran Singh "Efficient CBIR Using Color Histogram Processing. Signal \& Image Processing" : An International Journal(SIPIJ), Vol.2, No.1, pp. 94-112, March 2011.

[12] Ja-Hwung Su, Wei-Jyun Huang, Philip S. Yu, Fellow, and Vincent S. Tseng. "Efficient Relevance Feedback for Content-Based Image Retrieval by Mining User Navigation Patterns". IEEE Transactions on Knowledge and Data Engineering, Vol. 23, No. 3, pp. 360-372, 2011.

[13] Feng S., Lang C. and Xu D. "Localized content-based image retrieval using saliency-based graph learning framework". ICSP 2010 Proceedings, pp.1029-1032, 2010.

\section{Authors}

A. P.Anand was born on 16th Septemper 1987 in Madurai ,Tamilnadu, India. He received his B.E degree in Electronics and Communication Engineering at Karpagam College of Engineering, Coimbatore in 2009 and M.E degree in Communication Systems at SNS College of Engineering and Technology Coimbatore in 2011. He is working as Assistant Professor in the Department of Electronics and Communication Engineering, Velammal College of Engineering and Technology, Madurai. He has 3 years of teaching experience.

T. Ajitha doing her Final year B.E., in Electronics and Communication from Velammal College of Engineering and Technology, Madurai.
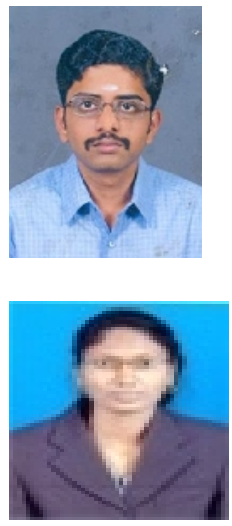
International Journal of Computational Science and Information Technology (IJCSITY) Vol.2, No.2, May 2014

M. Priyadharshini doing her Final year B.E., in Electronics and Communication from Velammal College of Engineering and Technology, Madurai.

M.G. Vaishali doing her Final year B.E., in Electronics and Communication from Velammal College of Engineering and Technology, Madurai.
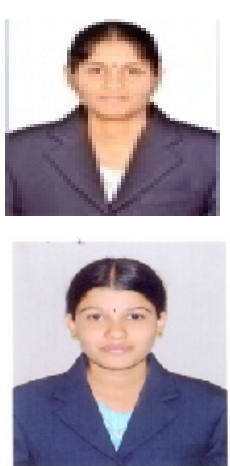